\title{
Mining Historical Traffic Data using Back Propagation Neural Network for Accurate Location Estimation
}

\author{
Rakesh Verma \\ Research Scholar, Acropolis Institute of \\ Technology \& Research, Indore, Madhya Pradesh, \\ India.
}

\begin{abstract}
The network technology is growing continuously and rapidly, their applications are also improved in the similar manner. Among these utilities and applications the location aware applications are also in high demand. These applications are required to evaluate more accurate positioning for improving the quality of applications services. Therefore in this presented work the techniques of location estimation are investigated. There are two main location estimation techniques are observed in literature, namely predictive and statistical estimation techniques.
\end{abstract}

Therefore, in this presented work the neural network based location approximation technique is evaluated. During implementation of neural network based predictive algorithm two key deficiencies are observed first the long training time and quality of training patterns. In order to improve both the issues in neural network the data pre-processing technique is proposed and implemented. The presented improvement helps in initializing neural network and frequent learning. Therefore, the neural network is effectively trained in less amount of time with higher accuracy.

The implementation of the proposed technique and traditional neural network technique is provided using NS2 network simulation environment and the neural network is implemented using JAVA environment. The performance evaluation of both the technique is given in terms of memory consumption, time consumption and predictive accuracy. According to the obtained results the performance of modified neural network is much higher than the traditional neural network. In addition of that the modified algorithm is able to train less amount of time as compared to the traditional neural network.

\section{Keywords}

Wireless Sensor Network, Neural Network, NS2, TOA and AOA.

\section{INTRODUCTION}

Wireless sensor network is a group of nodes organized into a cooperative network [1]. Each node comprises of processing capability (microcontrollers, CPUs, DSP chips etc), may include multiple types of memory, have a RF transceiver (usually with a single Omni directional antenna), have a power source (batteries, solar cells), and accommodating the various sensors and actuators. In such kind of cooperative network accurate position estimation is a complex task. The proposed work is an effort for estimating the mobile wireless sensor using the predictive methods. In this section of paper involves the overview of wireless sensor network, need of mobile node positioning, objectives, and finally the document organization overview.

Now in this world becomes small, due to advancement of communication technology. Quickly growing networks and

\author{
Abhay Kothari \\ Professor, Computer Science \& Engg. \\ Department, Acropolis Institute of Technology \& \\ Research, Indore, Madhya Pradesh, India.
}

modern techniques making revolution in this domain, according to the structure of network is categorized in two main domains first connection oriented and wireless oriented. While, according to their utility and applications the wireless communication is also illustrates in two parts, first short range or indoor communication and second broad range of outdoor communication.

A wireless sensor network is a distributed real-time system. A natural question is raised that how many solutions from distributed and real-time systems can be used in these new systems? Unfortunately yet very little work is applied in these new system and always a new solutions are often essential in all areas of the system [1]. Most of the earlier distributed systems research works on the following assumption like the systems are wired; powers is unlimited, not works on realtime, with a fixed set of resources, have user interfaces such as screens and mice, treat each node of the system as very significant and are location independent. Mobile positioning in wireless networks is mainly used for different kinds of application level services, such as security, monitoring, business and other similar domain application. In these applications required to find a particular place or person's location. It is also reasonable that future resource management algorithms may rely on position estimation and prediction. Therefore, there are mainly two approaches are available for position estimation. First using statistical method where node position is estimated using previous pattern of node mobility. And secondly the predictive method where based on previous mobility pattern data and extracted knowledge is used for a node position.

\section{PROPOSED WORK}

Wireless sensor is kind of cooperative network organization, which is frequently used in various different kinds of real world applications such as monitoring, navigation, and environmental conditions. Therefore, accurate location estimation is essential for these applications. Thus, here we intended to work with location estimation methods for finding the unique and accurate method for the position estimation. The complete study includes the following work:

1. Study different methods of location estimation: in this phase various techniques and methods are investigated, that are more promising for node position estimation for wireless mobile sensor networks.

2. Propose a new approach for location estimation: in this phase a new method is proposed and designed, that are providing the more accurate positioning information for wireless sensor nodes.

3. Implementation of the proposed method: the designed algorithm is implemented using the NS2 network simulator and TCL scripts, additionally, a recently used method is also implementing for the comparative study. 
4. Justification of the proposed study: the comparative analysis of both methods used for justifying the newly proposed solution.

In this research the main objective is to find the accurate location information using the data mining techniques. In such domain an essential contribution is placed by Anastasios Noulas et al [2] according to their description mobile location-based services are thriving, providing an unprecedented opportunity to collect fine spatio temporal data about the next places users visit. This source of data offers new possibilities to tackle established research problems on user mobility, but it also opens avenues for the development of novel mobile applications and other services. In this paper the problem of predicting the next venue a mobile user will visit, by exploring the predictive power offered by different facets of user behaviour. After that they propose a set of features that aim to capture the factors that may drive users' movements. Features exploit information on transitions between types of places, mobility flows between venues, and spatiotemporal characteristics of user check-in patterns. Further extend study in [2] by combining all individual features in two supervised learning models, based on linear regression and M5 model trees, resulting in a higher overall prediction accuracy of prediction. They find that the supervised methodology based on the combination of multiple features offers the highest levels of prediction accuracy. Therefore the predictive technique is able to predict more accurate positional information after analysing the historical mobility pattern. Thus in this presented work a new data mining based technique is evaluated for providing more accurate location information.

Problem Statement: Wireless location systems have received considerable attention and various different technologies have been proposed in the past few years. Wireless location techniques include angle of arrival, signal strength, time of arrival and time difference of arrival. TOA location scheme consumes the transmission time for a radio wave to move between the MS and a BS. The AOA scheme consumes an antenna array and a directive antenna to calculate the direction of arrival signal. The signal-strength scheme uses a known model to describe the path loss weakening with remoteness. Therefore the following issues are considered in the proposed study.

1. The available techniques are not much accurate and efficient.

2. Most of the techniques usage the additional hardware that may affect the estimation cost.

3. The predictive techniques are provides efficient and accurate results but the training time is not affordable.

In order to provide an optimum solution for location estimation in wireless sensor networks, neural network based technique is proposed. The neural network accepts historical data for analysis; this phase is also called the training of algorithm. During training the net weight is calculated and provided as the outcome of the neural network, after that the calculated outcome of neural network is compared with real outcome of input pattern. If difference between calculated outcome and real outcome is found then the neural network weights are adjusted for improving the network output. This phase is called the error propagation or error correction. When the neural network is trained with all the input samples than that is called trained model or network. This model is now able to predict the next location of the wireless sensor.
The neural network is an accurate technique for pattern recognition, but the neural network requires a significant amount of time for learning. Due to this that is not much appropriate when the quick learning is required. In addition of that during implementation input layer and hidden layers are mainly involved in results calculations. Therefore these two layers are denoted using a 2D vector. Furthermore when the network is initialized the random values are used to initialize the network. And using error propagation this vectors weights are adjusted. Due to adjustment of these random values a considerable amount of time is required.

Therefore in order to improve the learning technique of the neural network there are two key changes are incorporated in solution.

1. Changes in initialization phase: The neural network is initialized by initializing 2D vector with random values; these random values are between 0 and 1 . But the random initialization need more time for correction and adjustment. On the other hand initialization with 0 or 1 can result linearly. Therefore in order to enhance the learning time the following initialization algorithm is suggested for implementation.

\section{Table 1: Initialization Algorithm}

Input: $\mathrm{N}=$ number of input neurons

$\mathrm{H}=$ number of hidden layers

$D_{m, n}=$ input dataset

Output: initialized input and hidden layer

1. Create a $2 \mathrm{D}$ vector of size $\mathrm{NXH}$

2. $t h r=\sum_{b=1}^{n} \frac{D_{m, n}}{N}$

3. For $i=0 ; i<=n ; i++$

4. $\max \mathrm{A}=\max \left(D_{m, i}\right)$

5. $\min \mathrm{A}=\min \left(D_{m, i}\right)$

6. For $\mathrm{j}=0 ; \mathrm{j}<=\mathrm{m} ; \mathrm{j}++$

7. If $\mathrm{i}<\mathrm{H} \& \& \mathrm{j}<\mathrm{N}$

8. If $D_{i, j} \leq t h r$

9. $F_{i, j}=D_{i, j}$

10. $I_{i, j}=D_{i, j}$

11. End if

12. End if

13. End for

14. End for Return $F_{i, j}$

2. Improving the learning data: In the above given algorithm steps $I_{i, j}$ is used to initialize the neural network as the data is completed in neural network then it finish the initiation phase and for training purpose the filtered data $F_{i, j}$ is used for training input.

Simulation Architecture: Simulation architecture is presented by which the implementation work is performed. The present system includes three different data models that having their own methodology for data analysis and pattern discovery. 


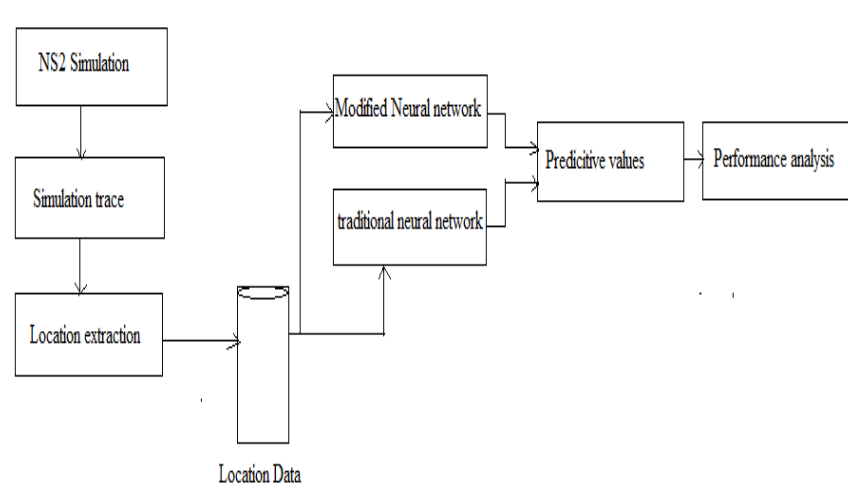

Fig 1: simulation architecture

The given model is described as:

1. NS2 simulation: in order to generate the location information first a wireless sensor network is configured with a number of network devices. These devices are known as the nodes in simulation. That is capable to visualize the network nodes and their mobility. This mobility is captured using generated trace files, with their changing positions in network.

2. Trace files: NS2 simulator is a discrete event simulator, during simulation generates trace files which contains different network parameters and their values. These trace file contains information about the node and their activities also. The node positions are extracted first using these trace files.

3. Location data: the trace files are used to extract location information, which is stored into a separate data file. This file is used as input for different neural network for training.

4. Modified neural network: in this module implements the modified neural network, which accepts the initial location for generating the next positions of network.

5. Traditional neural network: that implements the traditional neural network for generating new location information in network.

6. Predictive values: the generated values are stored separately for evaluating the performance of predictive algorithms.

7. Performance analysis: this phase includes the performance evaluation and comparative study between both implemented algorithms. The performance of these algorithms are is evaluated in terms of time and space complexity and the accuracy of the predictive algorithm.

\section{IMPLEMENTATION}

\subsection{Simulation Setup}

In this research provides the desired network configuration for simulation of proposed location estimation and simulation.

Table 2: Simulation Setup

\begin{tabular}{|l|l|}
\hline Simulation properties & Values \\
\hline Antenna model & Omni Antenna \\
\hline Dimension & 750 X 550 \\
\hline
\end{tabular}

\begin{tabular}{|l|l|}
\hline Radio-propagation & Two Ray Ground \\
\hline Channel Type & Wireless Channel \\
\hline No of Mobile Nodes & 10 \\
\hline Routing protocol & AODV \\
\hline Time of simulation & 10.0 Sec. \\
\hline
\end{tabular}

\subsection{Simulation Scenarios}

In order to simulate the effect of the predictive algorithms the following simulation scenarios are prepared.

\section{Simulation using traditional Neural network}

In this scenario the AODV routing algorithm is implemented for simulating the wireless sensor network with random mobility model. Using the trace the node movement is extracted for training and with respect to real time data the neural network's predictive performance of algorithm is evaluated.

\section{Simulation using Modified Neural network}

In this simulation the network is configured with the same routing protocol and the mobility model. And using the modified neural network the prediction performed, in this phase the performance of both the algorithm is also performed with the traditional neural network.

\section{RESULT ANALYSIS}

The performance evaluation of location estimation algorithms is performed in this research paper over different performance parameters.

Accuracy: The comparative accuracy of predictive algorithms is provided in this research study, that is a measurement by which the precision value between real time data and the predictive values are compared and how nearest the prediction is basically measured. That can be evaluated using the following formula.

$$
\text { Accuracy } \%=\left|\frac{\text { real position }- \text { predicted position }}{\text { real position }}\right| * 100
$$

The figure 2 shows the predictive accuracy of both the algorithms, the blue line shows the accuracy traditional neural network (TNN) and the performance of modified neural network (MNN) is given using red line. For representing the performance $\mathrm{X}$ axis shows the simulation time and the $\mathrm{Y}$ axis shows the accuracy in terms of percentage. In this experiment the similar training cycle is used. According to gained performance the accuracy of proposed neural network is much nearer to the real values. In addition of that for demonstrating the performance of algorithm for training figure 4.2 shows the accuracy of algorithms with increasing number of training cycle.

The given figure 2 shows the performance of both algorithms with increasing the number of training cycles. In this diagram the number of training cycles are given in $\mathrm{X}$ axis and the $\mathrm{Y}$ axis shows the accuracy improvement in terms of percentage. According to obtained results the modified neural network (MNN) is trained more efficiently as compared to the traditional neural network. 


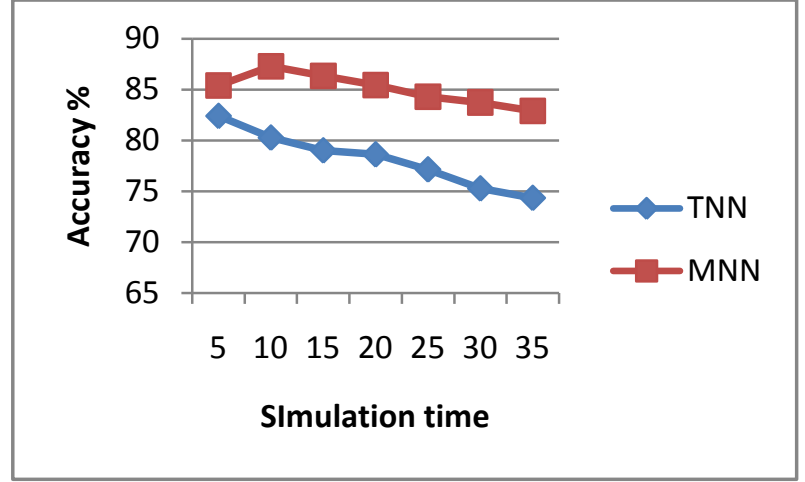

Fig 2: Accuracy

\section{Accuracy improvement:}

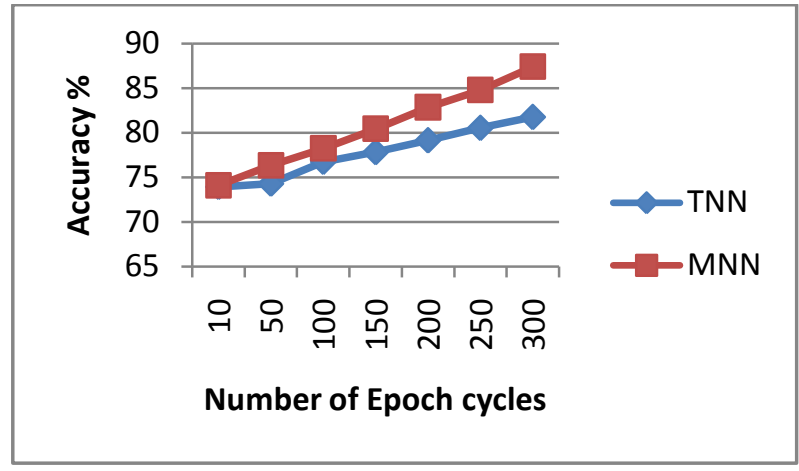

Fig 3: Accuracy improvement

Memory used: The amount of main memory required to train and predict values is known as memory consumed or space complexity. The space complexity of both algorithms TNN (traditional neural network) and MNN (modified neural network) is given using figure 4 . In this diagram $X$ axis shows the simulation time and the $\mathrm{Y}$ axis represents the amount of main memory consumed.

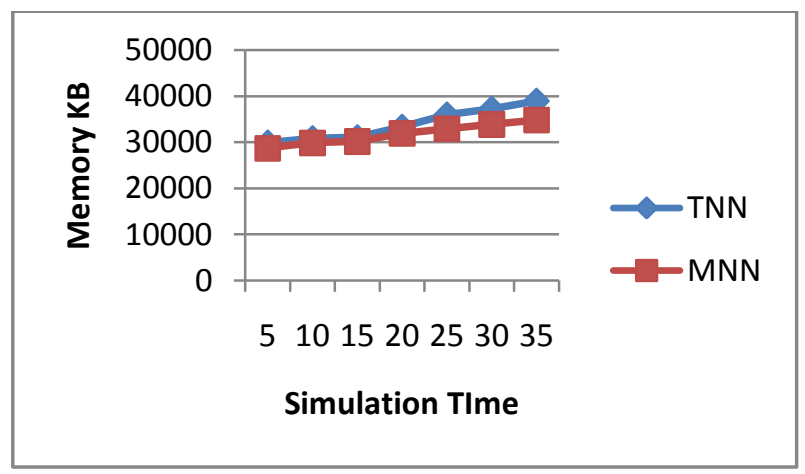

Fig 4: Accuracy improvement

The given figure 4 shows the space complexity of both the algorithm, according to the evaluated performance the memory consumption of the proposed modified neural network is much better than the performance of traditional algorithm. That is because the amount of patterns to learn is reduced during the training of neural network and with the pre-evaluated patterns the neural network is initialized.

Time Complexity: The amount of time required to perform training is known as time complexity of any algorithm. In neural network that is depends upon the number of training cycles consumed for appropriate learning of neural network algorithm. The time complexity of the proposed and traditional neural network is given using figure 5. In this diagram the blue line shows the time consumption of the traditional neural network and the red line shows the time consumption of the proposed modified neural network algorithm. For representing the results $X$ axis shows the number of experiments and the $\mathrm{Y}$ axis shows the time consumption of the time of algorithms. According to the obtained performance of both the algorithms the proposed modified algorithm is learned faster than the traditional algorithm.

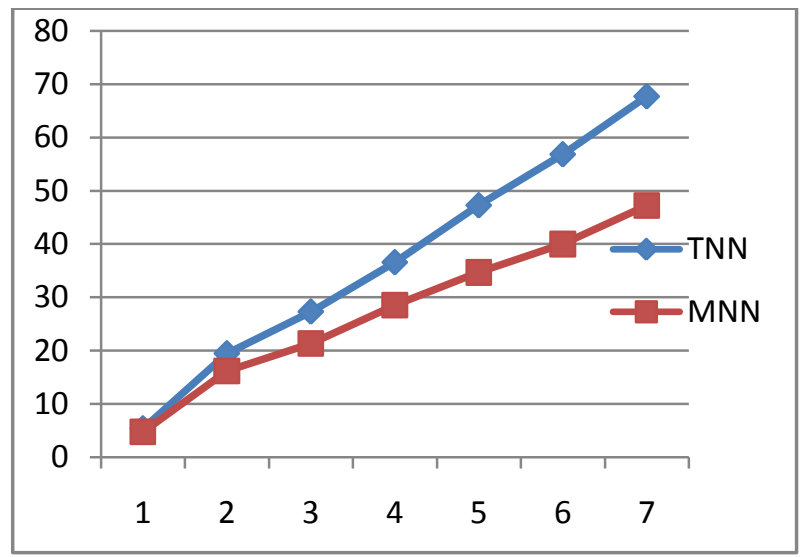

Fig 5: time complexity

\section{CONLUSION AND FUTURE WORK}

Now in these days various application level services are designed for targeting the location specific services. In addition of that these applications requires the location information for providing accurate data to the end client. Thus location estimation in such kind of applications is essential task. In this proposed work location estimation techniques for wireless sensor networks is investigated. According to the available literature there are two main streams for location estimation first using the statistical analysis and second using the predictive techniques. The main objective includes the study of predictive techniques for finding the accurate location of mobile sensors. This involves the study of data mining techniques for mobility pattern analysis and based on the discovered patterns the next sensor position estimation.

Therefore a neural network based predictive algorithm is developed and simulated using the NS2 network simulation environment. In order to find the more accurate location from neural network using less number of training cycles a mobility feature selection scheme is proposed and implemented with the neural network. The presented feature selection technique helps in initializing the neural network and mobility pattern discovery. Using the selected features, the neural network trained with limited amount of samples. In addition of that consumes less time to produce accurate location. According to the obtained performance with different experimentations the performance of proposed neural network is optimum and able to learn a significant mobility pattern in network. Therefore the presented technique is adoptable for predictive location estimation.

The presented location estimation technique is an accurate methodology among different kind of predictive techniques. That is optimized more for learning the mobility pattern in less computation cost (i.e. time and space complexity). In near future the proposed technique is enhanced more for improving the accuracy of prediction using regression analysis technique. 


\section{ACKNOWLEDGMENTS}

This work performs for our academic research only. It's not feasible for real time for all industrial application. The authors would like to thank the anonymous reviewers for their invaluable feedback.

\section{REFERENCES}

[1] J. Stankovic, "Wireless Sensor Networks", Department of Computer Science University of Virginia Charlottesville, Virginia 22904, 19 June 2006.

[2] A. Noulas, S. Scellato, N. Lathia and C. Mascolo, "Mining User Mobility Features for Next Place Prediction in Location-based Services", Proceedings of the 2012 IEEE 12th International Conference on Data Mining, pp. 1038-1043, 2012.

[3] T. Arampatzis, J. Lygeros and S. Manesis, "A Survey of Applications of Wireless Sensors and Wireless Sensor Networks", Proceedings of the $13^{\text {th }}$ Mediterranean Conference on Control and Automation Limassol, Cyprus, IEEE, pp. 27-29, June 2005.

[4] C. Song, K. Chung, J. Jung, K. Rim and J. Lee, "Localized Approximation Method Using Inertial Compensation in WSNs", New Challenges for Intelligent Information, SCI 351, pp. 247-255, 2011

[5] M. Z. Win, A. Conti, S. Mazuelas, Y. Shen, W. M. Gifford, D. Dardari and M. Chiani, "Network Localization and Navigation via Cooperation", IEEE Communications Magazine, pp. 56-62, May 2011.

[6] J. Wilson and N. Patwari, "A Fade-Level Skew-Laplace Signal Strength Model for Device Free Localization with Wireless Networks", IEEE Transactions on mobile computing, vol. 11, no. 6, June 2012.

[7] Z. Wang, W. Lou, J. Ma and H. Chen, "A Novel Mobility Management Scheme for Target Tracking in Cluster-Based Sensor Networks", International Journal of Distributed Sensor Networks, LNCS 6131, pp. 172186, 2010.

[8] R. Kulkarni and G. Venayagamoorthy, "Particle Swarm Optimization in Wireless Sensor Networks: A Brief Survey", IEEE Transactions on Systems, Man, and Cybernetics, Part C: Applications and Reviews, vol. 41, March 2011.

[9] A. Pal, "Localization Algorithms in Wireless Sensor Networks: Current Approaches and Future Challenges", Network Protocols and Algorithms, ISSN 1943-3581, vol. 2 , no. 1,2010

[10] P. Gao, W. Shi, W. Zhou, H. Li and X. Wang, "A Location Predicting Method for Indoor Mobile Target Localization in Wireless Sensor Networks", Hindawi Publishing Corporation International Journal of Distributed Sensor Networks, Article ID 949285, 30 January 2013.

[11] Z. Zhou, Z. Peng, J. Cui, Z. Shi and A. Bagtzoglou, "Scalable Localization with Mobility Prediction for
Underwater Sensor Networks", IEEE Transactions on Mobile Computing, vol. 10, 2011.

[12] S. Kar, J.M.F. Moura and K. Ramanan, "Distributed Parameter Estimation in Sensor Networks: Nonlinear Observation Models and Imperfect Communication", IEEE Transactions on Information Theory, Vol. 58, No. 6, June 2012

[13] J. Pan, S. Pan, J. Yin, Lionel M, N. Fellow and Q. Yang, "Tracking Mobile Users in Wireless Networks via SemiSupervised Co-localization", IEEE Transaction on Pattern Analysis and Machine Intelligence, vol. 34, no. 3, March 2012

[14] P. Gupta and S. S. Sutar, "Study of Various Location Tracking Techniques for Centralized Location, Monitoring \& Control System", IOSR Journal of Engineering (IOSRJEN) ISSN (e): 2250-3021, ISSN (p): 2278-8719, vol. 04, Issue 03, pp. 27-30, March 2014.

[15] N. Husted and S. Myers, "Mobile Location Tracking in Metro Areas: Malnets and Others", CCS'10, Chicago, pp. 4-8, October 2010

[16] F. Tsai, Y. S. Chiou and H. Chang, "A Positioning Scheme Combining Location Tracking with Vision Assisting for Wireless Sensor Networks", vol. 11, April 2013.

[17] M. R. Gholami, S. Gezici, and E. G. Strom, "Improved Position Estimation Using Hybrid TW-TOA and TDOA in Cooperative Networks", Copyright (c) IEEE, 2012.

[18] I. Guvenc, S. Gezici, and Z. Sahinoglu, "Fundamental Limits and Improved Algorithms for Linea LeastSquares Wireless Position Estimation", Copyright Mitsubishi Electric Research Laboratories Inc., TR2010108, September 2010.

[19] S. Singh, Shivangna and E. Mittal, "Range Based Wireless Sensor Node Localization using PSO and BBO and its variants", International Conference on Communication Systems and Network Technologies, IEEE, 2013.

[20] C. Luo, McClean, G. Parr, L. Teacy and R. D. Nardi, "UAV Position Estimation and Collision Avoidance using the Extended Kalman Filter", Copyright IEEE, 2013.

[21] V. Garg and M. Jhamb, "A Review of Wireless Sensor Network on Localization Techniques", International Journal of Engineering Trends and Technology (IJETT), vol. 4, April 2013.

[22] S.C. Liang, L. Liao and Y. Lee, "Localization Algorithm based on Improved Weighted Centroid in Wireless Sensor Networks", Journal of Networks, vol. 9, no. 1, pp. 183, January 2014.

[23] R. Kulkarni, A. Förster and G. Venayagamoorthy, "Computational Intelligence in Wireless Sensor Networks: A Survey”, IEEE Communications Surveys \& Tutorials, vol. 13, no. 1, First Quarter, 2011. 